Reprod. Nutr. Dévelop., 1986, 26 (2 B), 745-753.

\title{
Cinétique d'établissement de la microflore digestive chez le nouveau-né humain en fonction de la nature du lait
}

\author{
Marie-Christiane MOREAU, Michèle THOMASSON, R. DUCLUZEAU, P. RAIBAUD
}

avec la collaboration technique de Marie-Claude MULLER

Laboratoire d'Ecologie Microbienne,

I.N.R.A., 78350 Jouy-en-Josas, France.

Summary. Kinetics of bacterial colonization in the human newborn depending on the type of milk given.

The digestive tract of human infants, sterile at birth, is colonized by some bacterial genera within less than $48 \mathrm{~h}$. Among the factors involved in the implantation of a given bacterial genus, the type of milk fed plays a major role. We studied the development of fecal flora in 1 to 8-day old babies, either breast-fed or bottle-fed with humanized milk.

In breast-fed infants the microflora reached an equilibrium towards the age of 5 days and then hardly varied until the change of feeding.

Escherichia coli and Streptoccocus were established first and Bifidobacterium later. At the age of 5 days, the strictly anaerobic flora was exclusively composed of Bifidobacterium in $85 \%$ of cases. The development of other strictly anaerobic bacteria was repressed, particularly that of Bacteroides.

In infants receiving humanized milk, Escherichia coli lor sometimes other enterobacteria) also appeared very early in the digestive tract. However, the strictly anaerobic flora was either absent (40\% of cases) or composed of one or more genera (e.g. Bacteroides, Bifidobacterium, Plectridium).

These findings in human infants show that it is mainly the establishment of the strictly anaerobic flora which is affected by the type of milk fed.

\section{Introduction.}

A sa naissance, le nouveau-né n'héberge en général aucune bactérie. La colonisation bactérienne de son tube digestif est un phénomène très rapide, puisque $48 \mathrm{~h}$ après sa naissance, la flore fécale du nourrisson atteint $10^{8}$ bactéries $/ \mathrm{g}$ (Mata et Urrutia, 1971 ; Patte et al., 1979 ; Lejeune et al., 1981 ; Gracey, 1982). Cependant, toutes les souches bactériennes présentes dans l'environnement et principalement dans les flores vaginale et fécale de la mère, ne se développent pas dans le tube digestif du nouveau-né (Ducluzeau, 1983). On peut distinguer deux mécanismes de sélection qui interviennent chronologiquement lors de l'établissement de la microflore digestive. Un premier tri s'opère parmi les bactéries qui pénètrent dans le tube digestif : certaines vont seulement transiter, alors que d'autres pourront s'y développer (Patte et al., 1979). Les facteurs de ce tri sont encore très mal connus et semblent surtout liés à l'hôte et à son environnement 
(Lejeune et al., 1981). Puis un deuxième mécanisme intervient qui va permettre le développement de certaines souches bactériennes parmi toutes celles qui sont potentiellement capables de coloniser le tube digestif. La nature du lait semble alors le facteur le plus important de ce tri. Malgré les travaux publiés ces dernières années (Rotimi et Duerden, 1981 ; Stark et Lee, 1982 ; Benno et al., 1984), il en existe encore très peu traitant de l'influence de l'alimentation sur l'installation de la microflore digestive chez le nourrisson.

Le but de ce travail a donc été de déterminer la cinétique d'établissement de la microflore digestive durant la première semaine de vie chez des bébés qui étaient soit allaités par leur mère, soit nourris avec du lait de vache maternisé.

\section{Matériel et méthodes.}

1. Prélèvement des échantillons de fèces. - Les échantillons de selles ont été prélevés chez des enfants de la maternité de l'Hôpital Antoine Béclère (Clamart, France), dans le service du Professeur Papiernik. Tous étaient nés à terme par les voies naturelles, avec un test de Apgar $>9$, pesaient plus de $2,6 \mathrm{~kg}$ à la naissance et n'avaient pas reçu d'antibiotiques pendant leur séjour à la maternité. Leurs mères n'ont pas pris d'antibiotiques ni durant les 3 mois précédant l'accouchement, ni durant la période d'étude. Au total, 22 enfants nourris au sein et 11 nourris avec un lait de vache maternisé Gallia (Nursie 1er âge) ont été suivis. Des prélèvements quotidiens de selles ont pu être faits durant leur séjour à l'hôpital, soit 5 à 7 jours après leur naissance sur 12 enfants au sein et 6 au lait maternisé. Pour les autres enfants, seuls 2 à 3 échantillons de selles ont été obtenus durant leur première semaine de vie. Dans certains cas, des prélèvements ont pu être obtenus après le retour au domicile des mères et de leur bébé. La selle émise le matin par les enfants des différents groupes a été conservée à $4{ }^{\circ} \mathrm{C}$ jusqu'à l'analyse bactérienne, pendant un temps n'excédant pas $5 \mathrm{~h}$.

2. Analyse bactériologique des échantillons. - La technique de l'analyse différentielle quantitative a été utilisée (Raibaud et al., 1966). Brièvement, 0,5 à $1 \mathrm{~g}$ de selle a été homogénéisé à l'aide d'un ultraturrax dans 9 fois son poids d'eau stérile. Des dilutions décimales ont été faites dans le diluant I (Raibaud et al., 1966) et ensemencées dans différents milieux sélectifs ou non. Une goutte de la dilution au $1 / 100^{\circ}$ a été observée à l'état frais au microscope à contraste de phase (NIKON), dans le but de reconnaître, par leur morphologie, les genres bactériens les plus abondants dans l'échantillon. On s'assure ainsi que l'on cultive effectivement, dans les milieux choisis, les bactéries observées au microscope. Les milieux ensemencés ont été coulés dans des tubes de $8 \times 400 \mathrm{~mm}$ pour la numération des bactéries anaérobies strictes, résistantes à l'oxygène, puis incubés après solidification à $37^{\circ} \mathrm{C}$, pendant 7 jours. Pour la numération des bactéries anaérobies facultatives, les milieux ont été coulés en boîte de Petri.

Les entérobactéries ont été dénombrées sur le milieu " désoxycholate agar » (DIFCO). Les Streptococcus et autres cocci ont été dénombrés en aérobiose dans le milieu GAPTS 1 (Raibaud et al., 1961), rendu sélectif par addition d'azoture de sodium $0,003 \%$, ou encore en anaérobiose dans le milieu GAPTG (Raibaud et $_{10}$ 


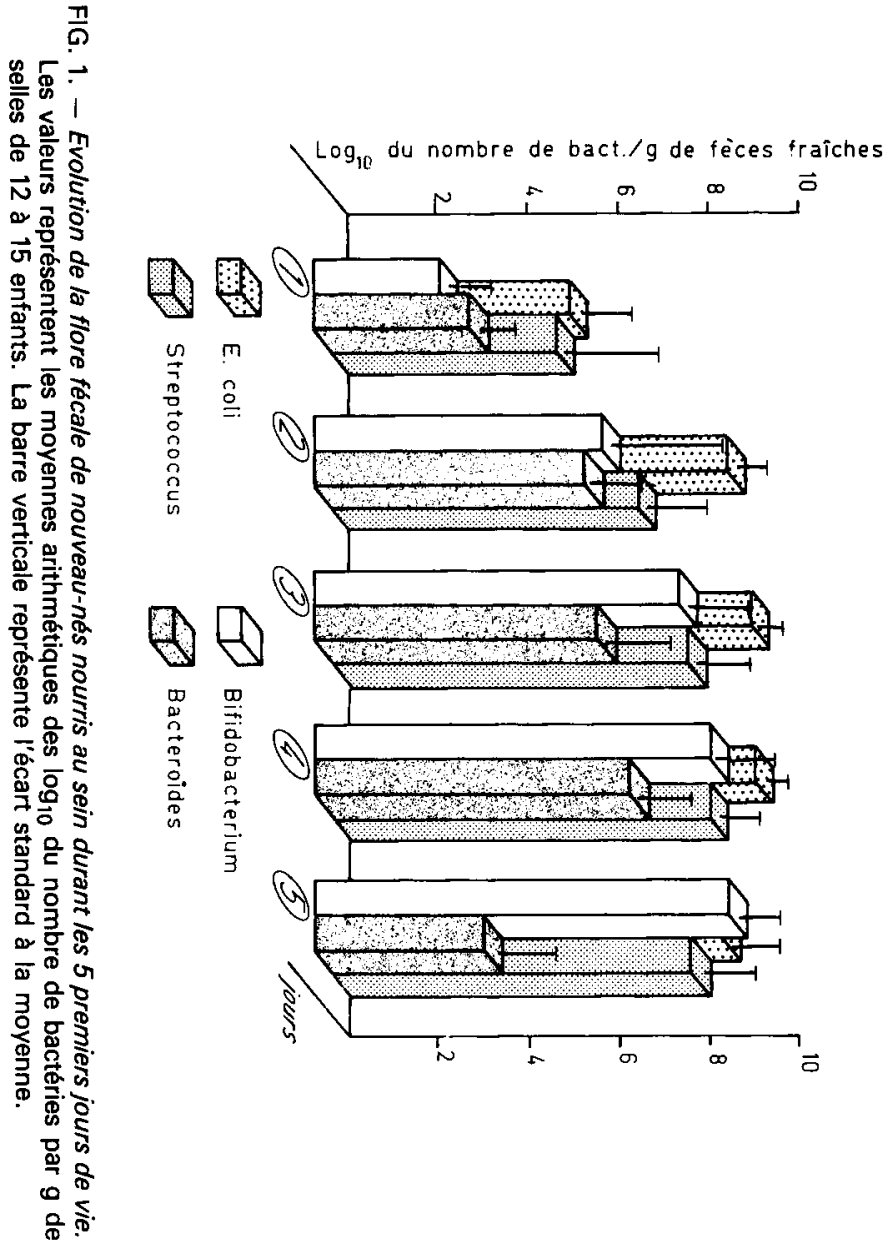



al., 1973) contenant de l'azoture de sodium à la même concentration. Ce dernier milieu permet également le dénombrement des Bifidobacterium et des Lactobacillus. Clostridium perfringens et les Bacteroides ont été dénombrés en anaérobiose dans le milieu B' (Sacquet et al., 1971) contenant 0,01\% de sulfate de néomycine (ICN). Les souches de Clostridium oxygène-tolérantes, les Plectridium et les Fusobacterium ont été dénombrées sur le milieu $D_{1}$ (Raibaud et al., 1966).

\section{Résultats.}

1. - Cinétique d'établissement de la microflore digestive en fonction de l'alimentation.

1.1. Enfants nourris au sein. - Les genres qui se développent dans les deux premiers jours sont anaérobies facultatifs (fig. 1) : Escherichia et Streptococcus. La flore anaérobie stricte, composée de Bifidobacterium et de Bacteroides se développe surtout à partir du $3^{e}$ jour. Entre le $3^{e}$ et le $5^{e}$ jour de vie, le nombre de Bifidobacterium augmente jusqu'à être, à 5 jours, le genre le plus abondant de la flore fécale. A ce moment, on constate que le nombre de $E$. coli a légèrement baissé et que le niveau de population de Bacteroides chute fortement. Au $5^{\mathrm{e}}$ jour de vie, la flore dominante, c'est-à-dire celle constituée des genres bactériens dont le nombre est supérieur ou égal à $10^{8} / \mathrm{g}$ de fèces, est composée de Bifidobacterium, de Escherichia et de Streptococcus.

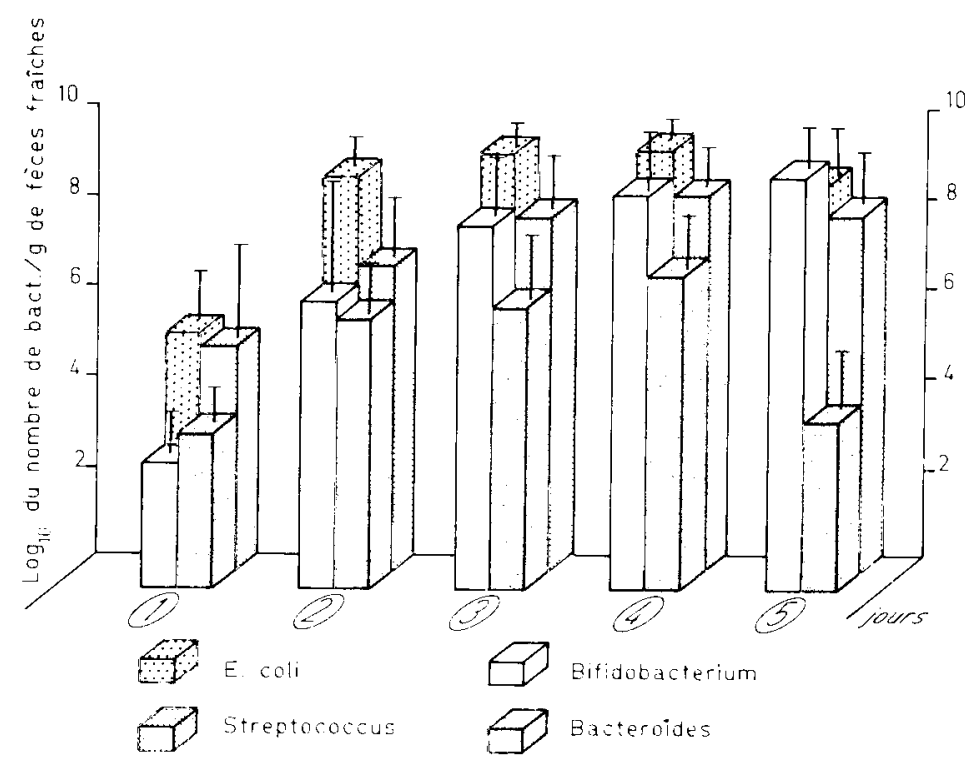

FIG. 1. - Evolution de la flore fécale de nouveau-nés nourris au sein durant les 5 premiers jours de vie. Les valeurs représentent les moyennes arithmétiques des $\log _{10}$ du nombre de bactéries par $\mathrm{g}$ de selles de 12 à 15 enfants. La barre verticale représente l'écart standard à la moyenne. 
On constate sur la figure 2 que l'équilibre microbien de la flore fécale présent à l'âge de 5 jours est stable puisqu'il est semblable chez les mêmes enfants âgés de 2 semaines.

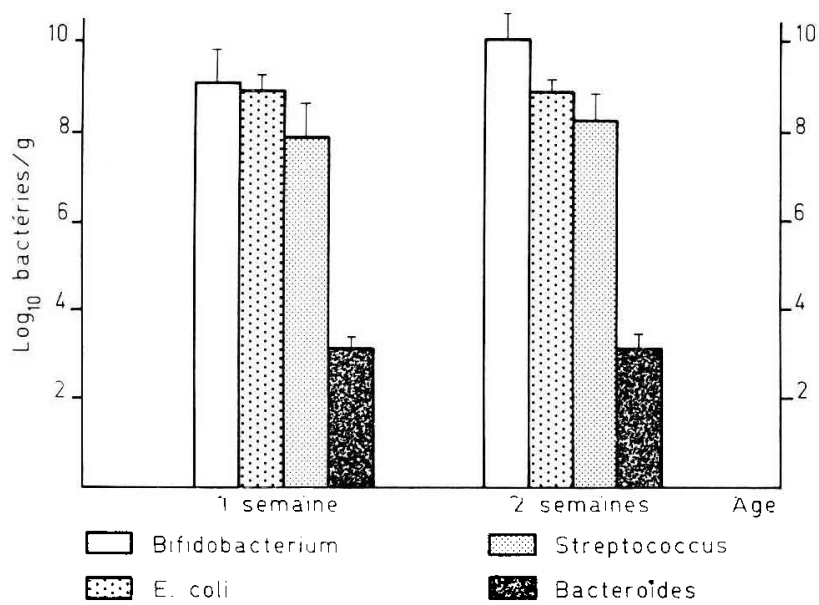

FIG. 2. - Evolution de la flore fécale de bébés nourris au sein en fonction de l'âge. Voir légende figure 1 (10 enfants).

1.2. Enfants nourris au lait de vache maternisé. - On voit sur la figure 3 que la cinétique d'établissement des bactéries anaérobies facultatives, durant les 2 premiers jours, est comparable à celle observée dans le groupe des enfants au

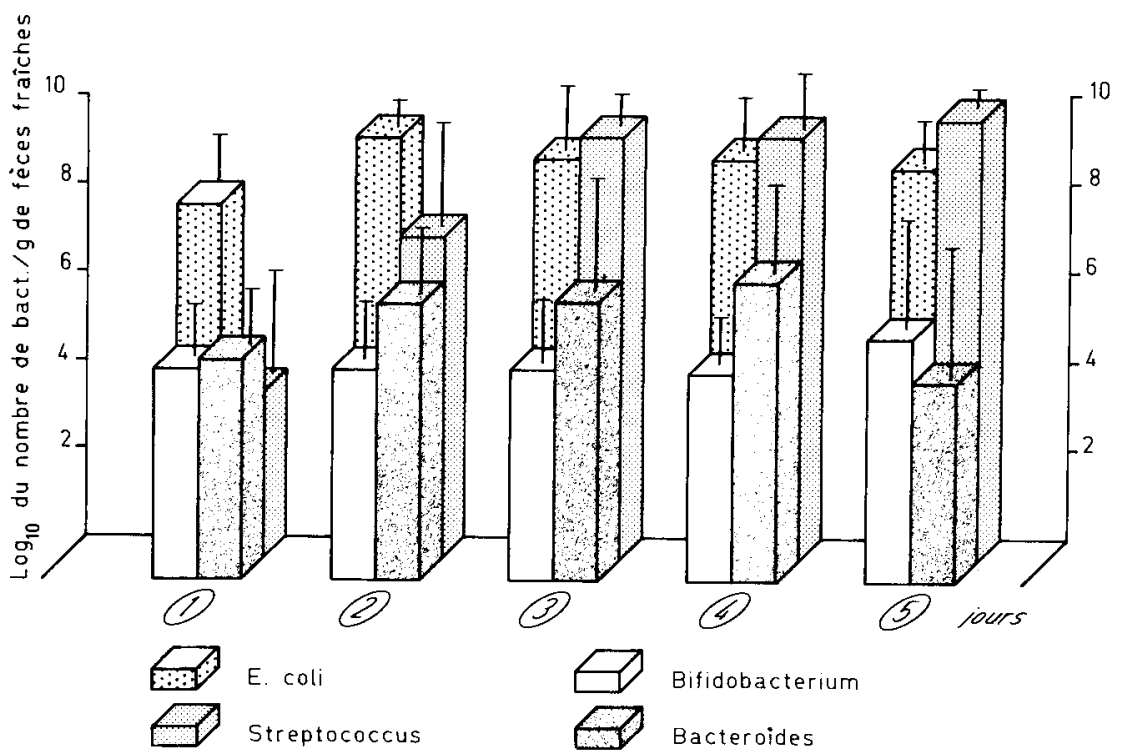

FIG. 3. - Evolution de la flore fécale de nouveau-nés nourris au lait maternisé, durant les 5 premiers jours de vie. Voir légende figure 1 ( 6 à 8 enfants). 
sein. Par contre, les niveaux de population des Bifidobacterium et des Bacteroides restent en moyenne faibles tout au long des 5 premiers jours de vie. A 5 jours, la flore dominante des fèces de ces enfants est constituée uniquement par Escherichia et Streptococcus, la flore anaérobie stricte restant à un niveau faible.

\section{2. - Comparaison des niveaux de population des genres anaérobies stricts de la flore fécale de nouveau-nés en fonction de leur alimentation.}

La flore anaérobie stricte présente à l'âge de 5 jours chez les enfants nourris au sein et ceux nourris au lait maternisé a été comparée. On voit (tabl. 1) que chez les enfants au sein, la flore anaérobie stricte n'est composée que du genre Bifidobacterium dans la grande majorité des cas étudiés ( $85 \%$ ). Une situation différente est observée chez les enfants nourris au lait maternisé. La flore anaérobie stricte est soit absente ou sous-dominante (40\% des cas), soit composée d'un ou de plusieurs genres bactériens : Bifidobacterium, Bacteroides, Clostridium, Plectridium, Fusobacterium. La nature de l'alimentation influence nettement le développement de la flore anaérobie stricte puisque (tabl. 2) les taux fécaux de Bacte-

\section{TABLEAU 1}

Comparaison des niveaux de population des genres anaérobies stricts de la flore fécale de nouveau-nés âgés de 4 à 6 jours, en fonction de la nature du lait.

\begin{tabular}{|c|c|c|}
\hline $\begin{array}{l}\text { Genres dominants } \\
\text { anaérobies stricts }\end{array}$ & Sein & $\begin{array}{l}\text { Lait de vache } \\
\text { maternisé Gallia }\end{array}$ \\
\hline Aucun & $5 \%(1 / 20)$ & $40 \%(4 / 10)$ \\
\hline \multicolumn{3}{|l|}{ Un seul } \\
\hline - Bifidobacterium & $85 \%(17 / 20)$ & $10 \%(1 / 10)$ \\
\hline - Bacteroides... & $5 \%(1 / 20)$ & $10 \%(1 / 10)$ \\
\hline 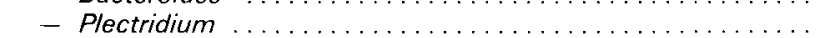 & 0 & $10 \%(1 / 10)$ \\
\hline - Fusobacterium ...... & 0 & $10 \%(1 / 10\}$ \\
\hline - Coques .......... & 0 & $10 \%(1 / 10)$ \\
\hline Plusieurs & $5 \%(1 / 20)$ & $10 \%(1 / 10)$ \\
\hline
\end{tabular}

\section{TABLEAU 2}

Evolution des niveaux de population des genres Bacteroides et Bifidobacterium dans les selles de nourrissons en fonction de leur âge et de la nature du lait.

\begin{tabular}{|c|c|c|c|c|}
\hline & \multicolumn{2}{|c|}{$\begin{array}{c}\text { Bacteroides } \\
\text { âge en jours }\end{array}$} & \multicolumn{2}{|c|}{$\begin{array}{l}\text { Bifidobacterium } \\
\text { âge en jours }\end{array}$} \\
\hline & $2-3$ & $4-5$ & $2-3$ & $4-5$ \\
\hline $\operatorname{Sein}(10)^{\mathrm{a}} \ldots \ldots \ldots \ldots \ldots \ldots \ldots$ & $8,4 \pm 0,4^{b}$ & $4,4 \pm 0,5$ & $8,8 \pm 0,5$ & $9,7 \pm 0,2$ \\
\hline Lait de vache maternisé $(8)^{a}$ & $5,8 \pm 2,4$ & $4,1 \pm 0,5$ & $4,7 \pm 1,4$ & $4,7 \pm 1,7$ \\
\hline
\end{tabular}

a : Nombre d'enfants ; b : Moyenne et écart à la moyenne des $\log _{10}$ du nombre de bactéries par $g$ de selles. 
roides et de Bifidobacterium sont élevés dès le $2^{e}$ jour chez les bébés au sein, alors qu'ils sont plus faibles chez les bébés nourris avec le lait maternisé. Au $5^{e}$ jour de vie, cette situation est inchangée chez les enfants nourris au lait maternisé, alors que chez les bébés allaités, on observe une répression du développement de Bacteroides.

\section{Discussion.}

Divers auteurs ont étudié soit la flore des enfants au sein (Mata et Urrutia, 1971), soit celle des enfants nourris au lait maternisé (Leclerc et Moriamez, 1980) ou encore celles des deux groupes d'enfants (Bullen et al., 1977 ; Long et Swenson, 1977 ; Benno et al., 1984). Dans le présent travail, la cinétique d'établissement de la totalité de la microflore digestive a été suivie quotidiennement durant la première semaine de vie dans les selles des mêmes enfants et l'effet de l'alimentation a pu être comparé chez des nourrissons nés et élevés dans des conditions d'environnement comparables.

Les analyses bactériologiques des selles ont permis de suivre l'évolution des niveaux de population de Escherichia, Streptococcus, Bacteroides et Bifidobacterium. Des cocci catalase positifs pouvant appartenir au genre Staphylococcus ont également été dénombrés dans certains de nos milieux. Cependant, les comptages de ces bactéries n'ont pas été rapportés dans les résultats, étant donné leur présence épisodique et à des taux peu élevés. Cette observation est en accord avec des études précédentes (Leclerc et Moriamez, 1980 ; Lejeune et al., 1981). Notons aussi que le genre Lactobacillus n'a été retrouvé en flore dominante que chez un enfant nourri au lait maternisé. Contrairement à ce qui est observé chez le raton, le souriceau, le porcelet, le veau, le genre Lactobacillus ne joue pas un rôle écologique important dans le tractus gastro-intestinal du nouveau-né humain, comme l'ont noté également Patte et al. (1979). Notre étude montre que les genres Streptococcus et Escherichia sont trouvés à des taux comparables chez tous les enfants des deux groupes. Ainsi, dans les 2 premiers jours de vie, la nature du lait ne jouerait aucun rôle sur le premier tri des genres bactériens qui s'opère chez le nouveau-né. Pourtant, l'enfant au sein a ingéré du colostrum riche en immunoglobulines de la classe des IgA et en lactoferrine dont l'effet bactériostatique sur E. coli a été démontré in vitro (Spik et al., 1978), mais pas in vivo (Moreau et al., 1983).

Puis vers l'âge de 2 à 3 jours s'installe la flore anaérobie stricte. Des différences sont alors observées entre les deux groupes d'enfants. Le lait maternel semblerait ainsi favoriser le développement de Bifidobacterium qui devient le seul genre anaérobie strict dominant à l'âge de 5 jours chez la quasi totalité des nourrissons suivis. Chez les enfants nourris au lait maternisé, la flore anaérobie stricte est très hétérogène, souvent absente ou composée de genres bactériens divers dont les taux sont fluctuants d'un jour à l'autre : Bacteroides, Plectridium, Clostridium, Fusobacterium... Ces genres se trouvent rarement chez les enfants au sein. Seul, Bacteroides semble pouvoir s'installer chez les enfants allaités par leur mère, mais son développement serait réprimé au cours des premiers jours de vie. Ce 
fait apparaît nettement dans les résultats rassemblés sur le tableau 2. Dans ce cas, les enfants retenus étaient ceux qui hébergeaient Bacteroides dans leurs selles à l'âge de 2-3 jours et dont nous possédions le prélèvement à l'âge de 4-5 jours. La répression de Bacteroides est moins évidente lors de la représentation cinétique de l'installation de la flore (fig. 1) où le critère du choix des enfants était différent : seuls les enfants dont nous avions tous les prélèvements entre l'âge de 1 à 5 jours ont été retenus et Bacteroides n'était pas présent chez tous les enfants, ce qui peut expliquer les valeurs plus faibles trouvées par rapport au tableau 2. Cette répression du développement de Bacteroides est en accord avec les travaux de Long et Swenson (1977) qui trouvent, à l'âge d'1 semaine, Bacteroides fragilis dans $22 \%$ des selles des bébés allaités contre $61 \%$ chez ceux nourris avec un lait maternisé. D'après Mata et al. (1971), ce n'est qu'au sevrage que la flore fécale des enfants nourris au sein s'enrichit de genres anaérobies stricts, autres que Bifidobacterium, pour atteindre progressivement le profil bactérien de la flore fécale de l'adulte (Ducluzeau et al., 1984). Ainsi, le facteur aliment pourrait jouer un rôle prépondérant dans ce deuxième tri qui s'opère parmi les bactéries anaérobies strictes de l'environnement.

Parmi les différentes hypothèses, la présence dans le lait de femme de substances favorisant le développement des bifides et, en particulier, l'espèce Bifidobacterium bifidum est celle qui a été retenue jusqu'à présent (Neut et al., 1980 ; Romond et al., 1980). Des essais ont en effet montré que certaines molécules appelées "facteurs bifidigènes », N acétyl-glucosamine, mucopolysaccharides, stimulent la croissance de B. bifidum in vitro (Beerens et al., 1980). Cependant, aucun résultat concernant l'implantation de bifides dans la flore fécale de nouveau-nés recevant des laits maternisés supplémentés avec ces facteurs bifidigènes n'a encore été publié. On peut ainsi supposer que l'action du lait de femme sur le développement des bifides in vivo puisse se faire par l'intermédiaire de l'hôte, en rendant le milieu digestif propice à l'implantation des bifides. Quoi qu'il en soit, les équilibres bactériens observés dans les selles des enfants étudiés diffèrent en fonction de leur alimentation.

La question se pose alors de savoir s'il faut favoriser la dominance des Bifidobacterium dans les selles des nouveau-nés. Récemment, Corthier et al. (1985) ont montré qu'une souche de $B$. bifidum inhibait la production de toxine par Clostridium difficile dans le tube digestif de souris à fiore contrôlée (souris gnotoxéniques), alors que le niveau de population de $C$. difficile était voisin de celui observé chez les souris témoins n'hébergeant pas $B$. bifidum. C'est la première démonstration d'un effet de $B$. bifidum sur l'activité métabolique d'une souche bactérienne potentiellement pathogène in vivo.

En conc/usion, il semble que la « maternisation » actuelle du lait de vache ne permette pas encore d'obtenir des séquences d'implantation des Bifidobacterium comparables à celles qu'on observe avec le lait de femme dans les selles des nouveau-nés. Il paraît important de s'attacher à résoudre ce problème dans la mesure où $B$. bifidum est susceptible de protéger le nouveau-né contre des bactéries potentiellement pathogènes lorsqu'elles peuvent se développer à un niveau élevé dans son intestin. 
Remerciements. - Nous remercions Monsieur le Professeur Gabilan, Chef du Service de Néonatologie, Madame Vial, Chef de Clinique au Service de Maternité "USI ", ainsi que tout le personnel de la Maternité de l'Hôpital Antoine Béclère, 92140 Clamart, pour nous avoir permis de réaliser ce travail.

Nous remercions également toutes les mères qui ont accepté, avec beaucoup de gentillesse, de collaborer à cette étude ainsi que la Société Gallia pour son aide financière.

\section{Références}

BEERENS H., ROMOND C., NEUT C., 1980. Influence of breast-feeding on the bifid flora of the newborn intestine. Am. J. clin. Nutr, 33, 2434-2439.

BENNO Y., SAWADA K., MITSUOKA T., 1984. The intestinal microflora of infants : composition of fecal flora in breast-fed and bottle-fed infants. Microbiol. Immunol., 28, 975-986.

BULLEN C. L., TEARLE P. V., STEWART M. G., 1977. The effect of " humanised " milks and supplemented breast feeding on the fecal flora of infants. Med. Microbiol., 10, 403-413.

CORTHIER G., DUBOS F., RAIBAUD P., 1985. Modulation of cytotoxin production by Clostridium difficile in the intestinal tracts of gnotobiotic mice inoculated with various human intestinal bacteria. Appl. environ. Microbiol., 49, 250-252.

DUCLUZEAU R., 1983. Implantation and development of the gut flora in the newborn animal. Ann. Rech. vet., 14, 354-359.

DUCLUZEAU R., LADIRE M., RAIBAUD P., 1984. Effet de l'ingestion de son de blé sur la flore microbienne fécale de donneurs humains et de souris gnotoxéniques receveuses et sur les effets de barrière exercés par ces flores à l'égard de divers micro-organismes potentiellement pathogènes. Ann. Microbiol. (Inst. Pasteur), 135 A, 303-318.

GRACEY M., 1982. Intestinal microflora and bacterial overgrowth in early life. J. Ped. Gastro. Nutr., 1, 13-22.

LECLERC H., MORIAMEZ J. C., 1980. Etude quantitative de la flore fécale de l'adulte et du nourrisson alimenté artificiellement. Path. Biol., 28, 217-226.

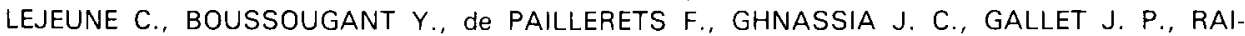
BAUD P., DUCLUZEAU R., 1981. Séquence d'installation de la flore intestinale du nouveau-né. Etude par analyse différentielle quantitative. Rev. Ped., 4, 223-242.

LONG S. S., SWENSON R. M., 1977. Development of anaerobic fecal flora in healthy newborn infants. J. Pediatrics, 91, 298-301.

MATA L. J., URRUTIA J. J., 1971. Intestinal colonization of breast-fed children in a rural aera of low socioeconomic level. Ann. N. Y. Acad. Sci., 176, 93-101.

MOREAU M. C., DUVAL-IFLAH Y., MULLER M. C., RAIBAUD P., VIAL M., GABILAN J. C., DANIEL N., 1983. Effet de la lactoferrine bovine et des IgG bovines données per os sur l'implantation de Escherichia coli dans le tube digestif de souris gnotoxéniques et de nouveau-nés humains. Ann. Microbiol. (Inst. Pasteur), 134 B, 429-441.

NEUT C., ROMOND C., BEERENS H., 1980. Contribution à l'étude de la répartition des espèces de Bifidobacterium dans la flore fécale de nourrissons alimentés soit au sein, soit par des laits maternisés. Reprod. Nutr. Dévelop., 20, 1679-1684.

PATTE C., TANCREDe C., RAIBAUd P., DuCluzeau R., 1979. Premières étapes de la colonisation bactérienne du tube digestif du nouveau-né. Ann. Microbiol. (Inst. Pasteur), $130 \mathrm{~A}$, 69-84.

RAIBAUD P., DICKINSON A. B., SACQUET E., CHARLIER H., MOCQUOT G., 1966. La microflore du tube digestif du rat. I. Techniques d'étude et milieux de culture proposés. Ann. Inst. Pasteur, 110, 568-590.

RAIBAUD P., GALPIN J. V., DUCLUZEAU R., MOCQUOT G., 1961. Studies on the bacterial flora of the alimentary tract of pigs. II. Streptococci : selective enumeration and differenciation of the dominant group. J. appl. Bact., 24, 285-306.

RAIBAUd P., GALPIN J. V., DUCLUZEAU R., MOCOUOT G., OLIVER G., 1973. Le genre Lactobacillus dans le tube digestif du rat. I. Caractères des souches homofermentaires isolées de rat holo- et gnotoxéniques. Ann. Microbiol. (/nst. Pasteur), 124 A, 83-109. 
ROMOND C., BEERENS H., NEUT C., MONTREUIL J., 1980. Contribution à l'étude de la maternisation des laits : influence in vitro du lait maternel, du lait de vache et de laits maternisés sur la croissance de Bifidobacterium. Ann. Microbiol. (Inst. Pasteur), 131 A, 309-314.

ROTIMI V. O., DUERDEN B. I., 1981. The development of the bacterial flora in normal neonates. J. med. Microbiol., 14, 51-62.

SACQUET E., RAIBAUD P., GARNIER J., 1971. Etude de la microflore de l'estomac, de l'intestin grêle et du caecum du rat conventionnel et de ses modifications à la suite de diverses interventions chirurgicales : anse aveugle jéjunale, déviations biliaires. Ann. Inst. Pasteur, 120, 501-524.

SPIK G., CHERON A., MONTREUIL J., DOLBY J. M., 1978. Bacteriostasis of a milk-sensitive strain of Escherichia coli by immunoglobulins and iron-binding proteins in association. Immunology, 35, 663-671.

STARK P. L., LEE A., 1982. The microbial ecology of the large bowel of breast-fed and formula-fed infants during the first year of life. J. med. Microbiol., 15, 189-203. 